\title{
MODELO DE INTERVENCIÓN ENFOCADO DESDE LA NIÑEZ AL PROTAGONISMO DE SU PROPIO APRENDIZAJE
}

Morera-Castro, María; Herrera-González, Emmanuel; Arguedas-Víquez, Rita; Fonseca-Schmidt, Héctor MODELO DE INTERVENCIÓN ENFOCADO DESDE LA NIÑEZ AL PROTAGONISMO DE SU PROPIO APRENDIZAJE

MHSalud, vol. 14, núm. 2, 2018

Universidad Nacional, Costa Rica

Disponible en: http://www.redalyc.org/articulo.oa?id=237054293003

DOI: https://doi.org/10.15359/mhs.14-2.3

Esta obra está bajo una Licencia Creative Commons Atribución-NoComercial-SinDerivar 3.0 Internacional. 


\title{
MODELO DE INTERVENCIÓN ENFOCADO DESDE LA NIÑEZ AL
} PROTAGONISMO DE SU PROPIO APRENDIZAJE

MODEL OF INTERVENTION FOCUSED ON CHILDHOOD AND THEIR PROTAGONISM

IN THEIR OWN LEARNING

MODELO DE INTERVENÇÃO ENFOCADO DESDE A INFÂNCIA AO PROTAGONISMO

DE SUA PRÓPRIA APRENDIZAGEM

\begin{abstract}
María Morera-Castro mmore@una.cr
Universidad Nacional, Costa Rica

Emmanuel Herrera-González emmanuel.herrera.gonzalez@una.cr

Universidad Nacional, Costa Rica
\end{abstract}

Rita Arguedas-Víquez ritaviquez@gmail.com

Universidad Nacional, Costa Rica

Héctor Fonseca-Schmidt tinfonse@yahoo.com

Universidad Nacional, Costa Rica

Recepción: 10 Noviembre 2017

Aprobación: 17 Enero 2018

DOI: https://doi.org/10.15359/mhs.14-2.3

Redalyc: http://www.redalyc.org/articulo.oa?id=237054293003

\section{Resumen:}

El objetivo de este artículo fue sistematizar la experiencia metodológica del Proyecto Psicomotricidad e Intervención desarrollado en la Universidad Nacional de Costa Rica. Este modelo de intervención apunta al fortalecimiento de las diferentes áreas del desarrollo humano en la población infantil de 2 a 7 años de edad, y sus ejes centrales: el desarrollo perceptual-motor y la auto-regulación. Como resultado de la aplicación de este modelo, el infante logró ser protagonista de su propio aprendizaje ya que, al ser expuesto a diferentes espacios y situaciones que, por medio del movimiento, la autonomía, el análisis y resolución de situaciones, logró aprender, afrontar y mejorar tareas diarias, destrezas y emociones, esto según la percepción de la persona encargada del infante, el personal del proyecto y los propios comentarios del infante. Se concluye que la creación de este modelo es una contribución para profesionales y, en general, para la comunidad interesada en la educación infantil y en su formación integral.

Palabras clave:

pedagogía, enseñanza, desarrollo motor, desarrollo del niño, ambiente educacional.

\section{Abstract:}

The purpose of this paper was to systematize the methodological experience acquired during the Psychomotricity and Intervention Project taught at Universidad Nacional, Costa Rica. This model of intervention aims at strengthening the different areas of human development in children ages 2 to 7, being perceptual-motor development and self-regulation the central axes. As a result of the implementation of this model children were able to become protagonists of their own learning. They were exposed to different spaces and situations that, through movement, autonomy, analysis and resolution of situations, they were able to learn, face and improve daily tasks, skills and emotions. The foregoing is according to the perception of the adult in charge of the child, project staff and the child's own comments. It is concluded that the creation of this model is a contribution for professionals and the general community interested in children's education and their overall formation.

Keywords: 
pedagogy, teaching, motor development, child development, educational environment.

Resumo:

O objetivo deste artigo foi sistematizar a experiência metodológica do Projeto Psicomotricidade e Intervenção desenvolvida na Universidade Nacional da Costa Rica. Este modelo de intervenção assinala o fortalecimento das diferentes áreas do desenvolvimento humano na população infantil de 2 a 7 anos de idade, e seus eixos centrais: o desenvolvimento perceptual-motor e a autorregulação. Como resultado da aplicação deste modelo, o infante conseguiu ser protagonista de sua própria aprendizagem, já que, ao ser exposto a diferentes espaços e situações que, por meio do movimento, a autonomia é análise e resolução de situações, conseguiu aprender, afrontar e melhorar tarefas diárias, destrezas e emoções, isto segundo a percepção da pessoa encarregada do infante, da equipe do projeto e dos próprios comentários do infante. Conclui-se que a criação deste modelo é uma contribuição para profissionais e, em geral, para a comunidade interessada na educação infantil e em sua formação integral.

Palavras-chave:

pedagogia, ensino, desenvolvimento motor, desenvolvimento da criança, ambiente educacional.

\section{Introducción}

El modelo que ofrece el Proyecto Psicomotricidad e Intervención, del Programa Psicomotricidad Infantil (PSICOMI), está inspirado en la problemática de la repitencia observada en el sector público en los niveles de preescolar y primer ciclo. El Ministerio de Educación Pública (MEP) estimó que, en el año 2000, hubo casi 45.000 repitientes en las escuelas de Costa Rica, de los cuales el 35 \% (15.627) cursaba el primer año escolar (Ministerio de Educación Pública [MEP], 2002).

En el Programa Estado de la Nación en Costa Rica, el cual presenta periódicamente informes con datos sobre la situación del país en distintos ámbitos, se señalan algunos datos a nivel educativo que resultan relevantes para esta investigación. Por ejemplo, en el Octavo Informe se indica que la deserción escolar se mantuvo relativamente estable con respecto a los años noventa. Sin embargo, la situación más crítica se ve representada en el hecho de que de cada 10 escolares que inician la primaria solo tres logran terminar la secundaria en el plazo establecido (Programa Estado de la Nación, 2002). Según el Sétimo Informe, en el 2005 se presentó una alta reprobación en primer grado, principalmente en la educación pública y en niños de zonas rurales (Programa Estado de la Nación, 2006). Además, en el Decimocuarto Informe se resalta que de cada mil escolares que ingresaron a primer grado de escuela en el 2007, solo 381 se graduaron de secundaria (Programa Estado de la Nación, 2008a). Por otra parte, en el Décimo Informe se indica que son los Centros de Educación y Nutrición (Cen-Cinai) los que atienden especialmente a menores de 6 años no escolarizados, con una cobertura de 79.104 personas (Programa Estado de la Nación, 2010).

A su vez, el Primer Informe del Estado de la Educación señala que la expansión de la educación preescolar podría ser un factor de incidencia en la disminución de reprobación en primer grado y que, por tanto, se ha producido una mejora en el rendimiento educativo y una disminución de la deserción escolar (Programa Estado de la Nación, 2005). No obstante, en el Segundo Informe del Estado de la Educación la tasa de reprobación en primaria mostró una tendencia creciente: de un $9 \%$ en el 2002 a un $11 \%$ en el 2006. Otro dato indica que escolares de primero y segundo ciclo carecen de hábitos de lectura, denotan bajos niveles de comprensión lectora y muestran serias deficiencias en la expresión escrita, lo que ocasiona un bajo rendimiento en la asignatura de Español (Programa Estado de la Nación, 2008b).

En el 2011, se señala en el Tercer Informe un aumento en la inversión a la educación pública ampliándose la cobertura en preescolar, y un aumento en la retención estudiantil. Además, hay un interés estatal por mantener la cobertura del nivel Interactivo II y de actualizar los programas de estudio de esta etapa de vida de acuerdo con los requerimientos actuales. Finalmente, se indica que es importante impulsar cambios que permitan una transición armoniosa de preescolar a primer grado con el firme propósito de reducir el fracaso escolar que se presenta en ese nivel (Programa Estado de la Nación, 2011). Un dato interesante es que la población menor a los cuatro años y tres meses actualmente queda fuera de la cobertura del Sistema Educativo Público (a pesar de que por mandato constitucional el Estado costarricense debe ofrecer servicios desde los 2 meses hasta los 6 años), condición que obligó a las familias a buscar alternativas en centros educativos privados, aun cuando la oferta fuera limitada, situación que se mantiene en la actualidad. 
Además, en este Cuarto Informe se asume la revisión y propuesta de los planes de estudio de los ciclos materno-infantil y transitorio y se indica que el $33 \%$ de esta población de 5 a 6 años provienen de hogares con clima educativo bajo (la escolaridad promedio de la totalidad de los miembros mayores de 18 años es inferior a seis años) y solo un $20 \%$ con clima educativo alto (un número de años de escolaridad igual o superior a doce). Asimismo, de manera general, esta población menor de 7 años presenta un débil desarrollo del área cognitiva y de lenguaje, que requiere ser fortalecido (Programa Estado de la Nación, 2013).

A lo largo de los diferentes informes del Estado de la Educación, es hasta el Cuarto Informe en donde se referencia la necesidad de incorporar en la educación preescolar la atención socioemocional, cognitiva y física dado el rol determinante que tiene la estimulación de estas áreas para el desempeño estudiantil, no solo en las edades tempranas, sino también en las edades de vida posterior. Previo a este informe, su enfoque se centró en el desarrollo del área cognitiva, social y emocional para el éxito escolar (Programa Estado de la Nación, 2013).

Para el 2015, en el Quinto Informe del Estado de la Educación, se evidencia la aprobación del nuevo programa de estudios del MEP en 2014, en el cual se destaca la integración de los dos ciclos preescolares y su articulación con el I Ciclo de primaria, además de la incorporación de la lectoescritura, una definición más precisa de las habilidades del perfil de salida al finalizar esta etapa del desarrollo y la creación de instrumentos para un mayor seguimiento docente, así como el establecimiento de un perfil del profesional. La cobertura de la educación preescolar sigue indicando insuficiencia y hasta un descenso en la misma: de 0 a 3 años esta cobertura es muy baja, en el interactivo Il el crecimiento es lento y en el ciclo de Transición se registró un descenso con respecto al 2013. Datos importantes señalan que hay diferencias significativas entre el porcentaje de asistencia de preescolares de hogares de mayor ingreso (70 \%) y los de bajo ingreso (40\%), brecha que se mantiene y hasta tiende al alza en comparación con el 2013. Otro dato a resaltar es que de esta población que asiste al preescolar público, más de un $50 \%$ de esta proviene de hogares pobres o en situación de vulnerabilidad. Cabe destacar que siguen siendo los CEN-Cinai los únicos programas que evalúan el desarrollo infantil (motora gruesa, motora fina, cognoscitiva, lenguaje, socioafectiva y hábitos de salud) para la población de 0 a 6 años, con una cobertura de 63.885 infantes beneficiados en el 2013 (Programa Estado de la Nación, 2015).

Como respuesta a la realidad recién descrita, el modelo del Proyecto Psicomotricidad e Intervención de PSICOMI surge como un tipo de intervención contestataria a las necesidades de atención que se presentan en la población infantil y en la niñez en Costa Rica. Por tanto, este artículo presenta la sistematización metodológica de la experiencia vivida en la construcción del modelo que emplea el Proyecto Psicomotricidad e Intervención impartido por el Programa de Psicomotricidad Infantil en la Escuela de Ciencias del Movimiento Humano y Calidad de Vida de la Universidad Nacional, Costa Rica, en la población infantil de 2 a 7 años de edad.

\section{Marco referencial}

Para entender la génesis del modelo empleado por el Proyecto Psicomotricidad e Intervención, es necesario hacer referencia a aspectos relevantes que dieron origen a la fundamentación de PSICOMI. Primeramente, se realizó un diagnóstico que se llamó Sistema Peques (Herrera, Araya, Fernández, Morera y Fonseca, 2015), una batería de pruebas psicomotrices diseñadas y estandarizadas que tiene como propósito detectar cuando los niños y las niñas son posibles candidatos y candidatas a tener un bajo rendimiento escolar, y, por ende, la eventualidad de repitencia en los primeros años de educación. Este diagnóstico se centró en la evaluación del desarrollo motor, (Woodburn, Rodríguez y Boschini, 1997), específicamente en la coordinación corporal (Woodburn, Camacho, Boschini, Fernández y Rodríguez, 1991), los problemas de aprendizaje (Woodburn y Boschini, 1997), la imagen corporal (Woodburn, Boschini y Fernández, 1997), las prueba para configuraciones (Woodburn, 1997a) y la madurez intelectual (Woodburn, 1997b).

A partir del Sistema Peques, se inició con intervenciones a través de la estimulación perceptual-motora (paradigma de la psicomotricidad) como forma de trabajo (Woodburn, Sisfontes y Fernández, 1998). Esta intervención se realizó a niños y niñas que al ser evaluados con el Sistema Peques presentaron situaciones que podrían influir en su rendimiento escolar. Esta intervención cumplió con dos aspectos importantes: el 
primero fue crear una metodología de trabajo a través del descubrimiento guiado y, por otro lado, contar con el apoyo familiar durante las sesiones de juego y trabajo (Herrera, Delgado, Fonseca y Vargas, 2012).

Derivado de esta experiencia, el programa PSICOMI ha realizado la aplicación de pruebas e intervenciones psicomotrices, pruebas psicopedagógicas y pruebas en edades tempranas, que determinan posibles dificultades a nivel académico y predicen un posible éxito o fracaso del niño o la niña en sus primeros tres años de educación primaria. A su vez, ha elaborado informes para familiares e instituciones educativas, ha efectuado intervenciones para fomentar estilo de vida saludable en niños y niñas, e investigaciones en las áreas temáticas que atañen al programa y a las necesidades del país en cuanto a la población infantil, niñez y adolescencia. Algunas investigaciones a citar son: Álvarez (2015, 2016a, 2016b); Herrera, Araya, Fernández, Morera y Fonseca, (2015); Herrera, Delgado, Fonseca y Vargas (2012); Herrera-González, Morera-Castro, Vargas-Araya, Fonseca-Schmidt y Vargas-Ramírez (2017).

\section{Metodología de la sistematización del proceso de construcción del Modelo de Intervención del Proyecto Psicomotricidad e Intervención.}

Desde el año 2008 hasta el 2012 se realizó en la Escuela de Ciencias del Movimiento Humano y Calidad de Vida de la Universidad Nacional un proyecto cuyo objetivo era beneficiar a niños y niñas en su rendimiento académico por medio del juego o del movimiento humano. Mucha de la población asistente en esta etapa estaba compuesta por escolares que eran referidos por maestros de escuela, centros de salud o casos particulares con problemáticas de maduración. Durante estas sesiones, la metodología utilizada fue el descubrimiento guiado, el cual consiste en plantear preguntas para que los niños y las niñas de manera exploratoria lograran descubrir el movimiento o el concepto solicitados por sí mismos. Posterior a las sesiones de trabajo, las cuales tenían una duración de todo un año, se solicitaban las notas de cada niño o niña y se cotejaba su aprobación o no del curso lectivo.

Una vez concluida esta primera etapa de investigación, el proyecto abrió sus puertas al público en general, bajo la modalidad de venta de servicios, y fue en este momento en el cual se realizó una reflexión de la pertinencia del trabajo que se venía realizando y se efectuaron mejoras que beneficiaran no solo a la niñez sino también a sus familias. Aunado a esto, la llegada de una nueva metodología de trabajo alentó a las personas participantes del proyecto a querer realizar innovaciones en este. Es así como, en el año 2014, se inicia dentro del Programa PSICOMI, un proyecto denominado Psicomotricidad e Intervención, que permitió ampliar los espacios de interacción entre el infante y sus pares, implementar nuevas guías metodológicas, generar nuevos ambientes de aprendizaje e involucrar a las familias en la formación y desarrollo de sus hijos e hijas. Como parte de este proyecto, se creó un modelo de intervención, el cual apunta al fortalecimiento de las diferentes áreas del desarrollo humano (social, emocional, físico, motor y cognitivo) en población infantil de 2 a 7 años de edad, con dos ejes centrales de acción: el desarrollo perceptual-motor y la auto-regulación (ver Figura 1).

Es importante señalar que se utilizaron varias técnicas y estrategias para la construcción de este modelo. Primeramente, se realizó una revisión bibliográfica de los principales referentes teóricos a nivel motor, perceptual y de aprendizaje existentes, los cuales contribuyeron en la integración de las experiencias prácticas previas del Programa PSICOMI. Una segunda fase consistió en el análisis de lo que se había realizado en el Programa PSICOMI y las necesidades actuales de la población infantil en Costa Rica. Lo anterior propició la creación de un modelo con una base en fundamentos teóricos, teorías de aprendizaje y experiencias profesionales vividas. Todo esto se llevó a cabo en secciones de trabajo participativas y reflexivas entre las personas que participan en el Programa. Finalizado el modelo, se procedió a la elaboración de pautas metodológicas que permitieran poner en práctica el modelo en el proyecto Psicomotricidad e Intervención. A partir de esto, se determinó qué aspectos del desarrollo humano se trabajaría, según la etapa de vida y el modelo del Proyecto Psicomotricidad e Intervención (ver Tabla 1). 


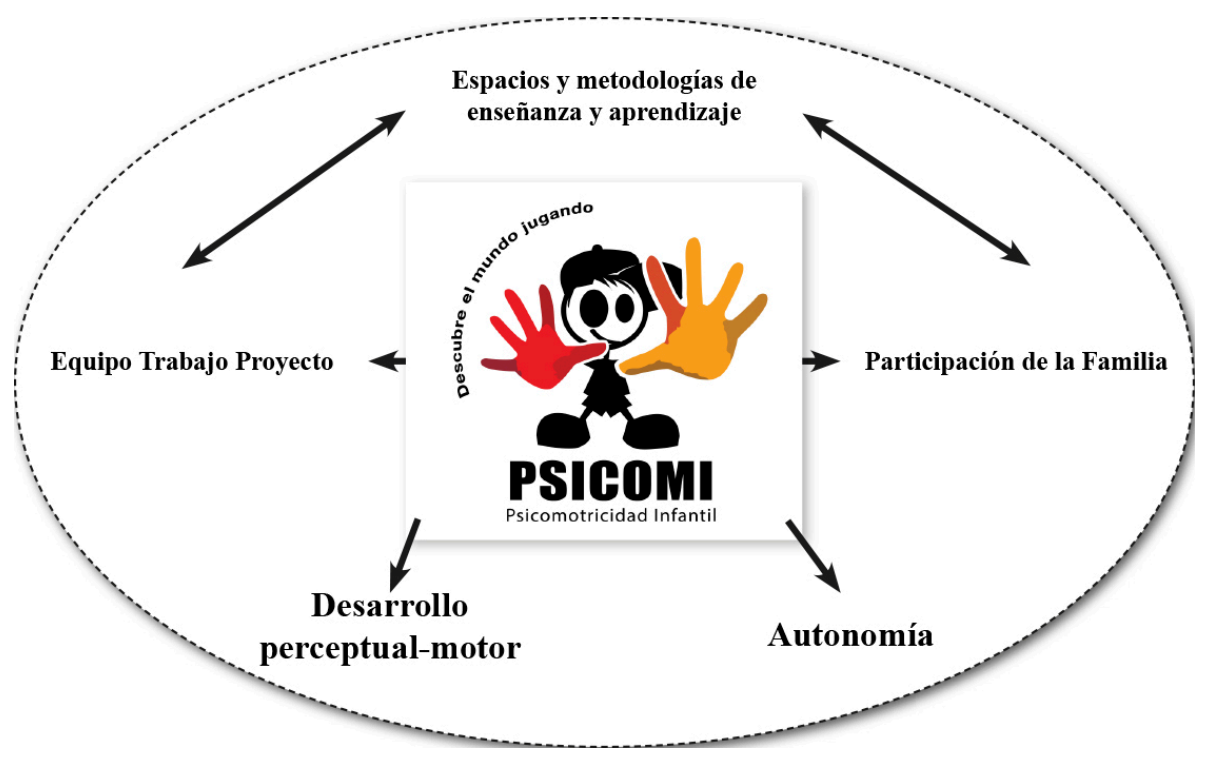

Figura 1

Modelo del Proyecto Psicomotricidad e Intervención Nota: Elaboración propia

\section{Fundamentos teóricos del Modelo de Intervención del Proyecto Psicomotricidad e Intervención}

Este modelo tiene como fundamentos teóricos el descubrimiento guiado y el Clima de Ejecución Motivacional (en inglés, Master Motivational Climate), los cuales permiten un modelo creado desde la niñez para la niñez, como principales protagonistas de su aprendizaje.

\section{Descubrimiento guiado}

El descubrimiento guiado es un estilo de enseñanza propuesto por el profesor Muska y Mosston, quien lo conceptualiza como una forma para identificar, aclarar y moderar el comportamiento del docente durante el proceso (Mosston y Ashworth, 1986). Los estilos de enseñanza influyen en la actuación del educador brindándole diferentes posibilidades para que el proceso de enseñanza se dé en función de las necesidades de los estudiantes (Fernández Rivas y Espada Mateos, 2016). El descubrimiento guiado consiste en una relación exclusiva entre el profesor y el estudiante, donde la secuencia de preguntas del primero conlleva a respuestas del segundo, permitiendo construir conocimiento. Este estilo tiene una anatomía de tres momentos, los cuales son denominados preimpacto, impacto y postimpacto. En el preimpacto, el profesor confecciona la clase y formula las preguntas a realizar; el impacto es la clase y es donde los estudiantes experimentan y descubre los movimientos o conceptos; mientras que el postimpacto es la retroalimentación. En este estilo, el feedback es único, ya que de algún modo es intrínseco y se da paso a paso, luego de cada actividad, con el refuerzo brindado por los demás (Mosston y Ashworth, 1986).

Para poner en práctica este estilo, es necesario que el profesor esté dispuesto a cruzar el umbral de descubrimiento, confiar en la capacidad de sus estudiantes y tener paciencia para esperar la respuesta adecuada (Mosston y Ashworth, 1986).

\section{Master Motivational Climate (Clima de ejecución motivacional)}

El Master Motivational Climate es un programa diseñado con el fin de que las personas profesionales en educación física cuenten con un modelo de desarrollo de destrezas motoras progresivo y apropiado para todo el estudiantado. Este programa tiene como base epistemológica la teoría social cognitiva, la cual incorpora una serie de modelos motivacionales: autoeficacia, competencia percibida y varias perspectivas orientadas al logro de objetivos que permiten explicar como la persona enfrenta, participa en o responde para lograr actividades o adquirir comportamientos. Por esto, el clima motivacional se establece cuando la persona docente estructura un proceso instruccional completo que permita la convergencia de ciertas 
metas basado en el contexto de un ambiente de aprendizaje (Ames, 1992a y 1992b). Es una metodología desarrollada prioritariamente para el enriquecimiento de los ambientes de juego, brindando múltiples oportunidades de aprendizaje para el dominio propio de los niños y las niñas, por lo que se fomenta y cultiva la independencia, se promueve su desarrollo integral. Asimismo, se impulsa el aprendizaje de destrezas tales como destrezas motoras fundamentales, representación numérica, reconocimiento de colores o desarrollar una perspectiva histórica de los eventos. También fomenta el desarrollo afectivo y emocional, las destrezas del lenguaje, entre otros (Wall y Rudisill, 2002; Wall y Rudisill, 2004; Valentini y Rudisill, 2004).

Este programa de intervención se basa en estructurar una clase tomando como elementos seis dimensiones, las cuales se conocen como TARGET: tarea, autonomía, reconocimiento, agrupamiento, evaluación y estructuras de tiempo. La tarea consiste en desarrollar una variedad de actividades que desafíen a la persona según su nivel de destreza y que a la vez le permitan una participación activa. La autonomía se enfoca en involucrar al estudiante en la toma de sus propias decisiones y en roles de liderazgo, permitiendo un desarrollo de la autogestión y la destreza de la autoevaluación. El reconocimiento se enfoca principalmente en potencializar la autoestima de cada estudiante, a través del reconocimiento y progreso individual positivo garantizando la igualdad de oportunidades y evitando las comparaciones sociales. El agrupamiento se debe usar de manera flexible, heterogénea, utilizando criterios de acuerdo al progreso, mejoramiento y ejecución, y propiciando arreglos de agrupamiento múltiples, así como el trabajo individual. La evaluación va enfocada en tres aristas: la autoevaluación, coevaluación y la realimentación o feeback por pares y por parte de la persona docente. La estructura del tiempo debe ser flexible, que propicie oportunidades y tiempo para el mejoramiento, donde el estudiantado pueda establecer su propio trabajo, tareas y prácticas y permita las instrucciones individuales (Valentini, Rudisill y Goodway, 1999a y 1999b).

Teorías del aprendizaje que fundamentan el Modelo de Intervención del Proyecto Psicomotricidad e Intervención Una de las teorías que sustenta este modelo es el aprendizaje por descubrimiento, de Jerome Bruner. Este consiste en el descubrimiento progresivo por parte del infante de lo que desea aprender. El descubrimiento guiado tiene su punto medular en la exploración motivada por la curiosidad, lo cual requiere que la persona facilitadora tenga el objetivo muy claro, elabore estrategias adecuadas y facilite material potencialmente significativo que propicie la exploración y la investigación a partir de las propias ideas y experiencias en la población participante (Bruner, 1995).

Relacionado con lo anterior, el aprendizaje significativo, de David Ausubel, otra teoría en la cual se basa el modelo, conlleva un proceso constructivo interno, autoestructurante (subjetivo y personal), que contiene un importante componente afectivo, en el que el autoconocimiento, la disposición por aprender y las expectativas propician la creación de nuevas estructuras cognitivas mediante estrategias que vinculan los aprendizajes previos con nueva información por aprender. De igual manera, el aprendizaje significativo se ve facilitado gracias a la mediación e interacción social y cooperativa con otros: es un proceso de reconstrucción de saberes culturales que a su vez implica un proceso de reestructuración interna de esquemas dependiendo del nivel de desarrollo cognitivo, emocional y social de cada persona o familia. De acuerdo con esta teoría, la persona aprendiz debe trabajar con tareas auténticas y significativas, y necesita aprender a resolver problemas con sentido, mediante apoyos que conduzcan a la construcción de puentes cognitivos entre lo nuevo y lo familiar con materiales potencialmente significativos (DíazBarriga y Hernández-Rojas, 2010).

La educación es un instrumento decisivo para el desarrollo de las personas, por eso debe ser comprendida y diseñada desde esta visión; se trata de educar para que las personas aprendices desarrollen capacidades que las hagan competentes en el contexto social en el que se desenvuelven. Este principio de la teoría del aprendizaje cooperativo y cultural de Lev Vigostky se asume en el modelo, a través de un diagnóstico previo que permita conocer la zona de desarrollo real de cada infante con el fin de generar actividades en diferentes momentos que le permitan al infante bajo la guía de otros llámense persona facilitadora, persona encargada, pares o persona compañera más capaz- alcanzar su zona de desarrollo próximo o potencial, dando cabida al aprendizaje guiado y cooperativo en el que se establece un aprendizaje dialógico entre las personas actoras en el proceso (Trilla et al., 2007). 
Otro propósito de este modelo es potenciar la autorregulación y la autonomía del infante en su proceso de aprendizaje, entendiendo que ser autónomo supone dominar un conjunto amplio de estrategias para aprender o ser capaz de tomar decisiones intencionales, conscientes y contextualizadas (Monereo et al, 2008). En este sentido, el proyecto Psicomotricidad e Intervención propone al infante una diversidad de actividades motrices que elige a partir de su autoconocimiento y su nivel de desarrollo con las que, progresivamente y con la guía de la persona facilitadora, compañero o par, irá alcanzado procesos más complejos de desarrollo en todas sus áreas, logrando autorregularse y ser una persona autónoma en su vida.

\section{Metodología de la aplicación del Modelo de Intervención del Proyecto Psicomotricidad e Intervención \\ Este proyecto se desarrolla los sábados en horas de la mañana, durante tres horas, con la participación activa tanto de la población infantil como de sus familiares o personas encargadas. Las actividades están guiadas por profesionales en Ciencias del Movimiento Humano y áreas afines, y estudiantes de la Escuela de Ciencias del Movimiento Humano y Calidad de Vida, de la Universidad Nacional, Costa Rica.}

Durante toda la mañana, las familias van pasando por tres diferentes momentos de aprendizaje: acuático, salón y aire libre, el cual es representado en la Figura 2. En los momentos: acuáticos y al aire libre, la participación de la familia o la persona encargada es fundamental, ya que proporcionan un ambiente de seguridad para esta población. En el caso del momento en el salón, la familia o la persona encargada no participa y permite que el o la infante, junto con los pares y las personas facilitadoras del proyecto, tengan un momento de interacción e integración. Además, hay de un espacio de socialización para merendar.

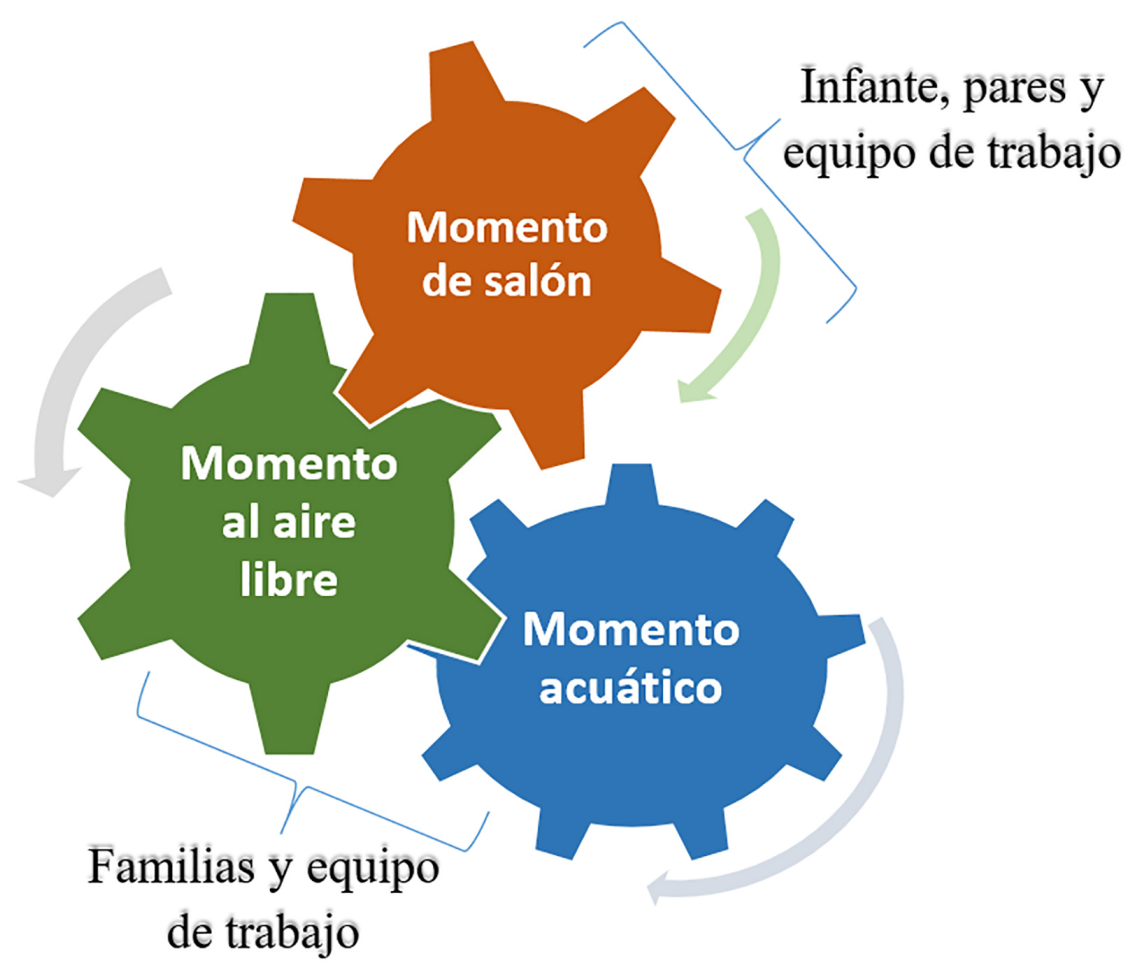

Figura 2

Espacios de aprendizaje del Modelo del Proyecto Psicomotricidad e Intervención Nota: Elaboración propia

De manera general, las actividades al aire libre utilizan la metodología del descubrimiento guiado, donde se promueven diferentes actividades orientadas a la búsqueda de la sensibilización, la exploración, el trabajo en equipo, la apreciación y amor hacia la naturaleza, el entorno, los seres humanos, animales o cualquier ser viviente, así como el desarrollo de la motora gruesa, dando la posibilidad a las personas participantes de poder contar con elementos importantes en la promoción de estilos de vida saludables. 
Las actividades acuáticas se realizan en la piscina, sin embargo, si no se cuenta con ese recurso, se planifican actividades donde el agua es su principal protagonista. En este momento se promueve la autorregulación, el desarrollo básico de destrezas acuáticas y la sobrevivencia en el agua, así como aspectos emocionales y sociales, por lo que la participación activa de la persona encargada y la familia se convierte en uno de nuestros pilares.

En el momento de salón, la metodología Master Motivational Climate permite el contacto con sus pares y las personas facilitadoras del Programa, en el cual se ponen en juego los factores emocionales, sociales, cognitivos y motrices. Debido al espacio con que se cuenta, únicamente los niños de estimulación temprana, el grupo 2 y 3, y 4 y 5 años participan en el laboratorio. En el caso del grupo de 6 y 7 años se les brinda un espacio como el gimnasio o canchas multiuso para potencializar el espacio de aprendizaje.

Como parte de la metodología de trabajo de la sala de psicomotricidad, se brindan indicaciones básicas de seguridad y se organiza la sala con materiales de diferentes tipos, tamaños y formas (con un objetivo de aprendizaje específico), colocados estratégicamente con el propósito de que esta población interactúe entre sí y con el ambiente. Las actividades por si solas son variadas, para que esta población tenga la oportunidad de elegir según sus capacidades. Las intervenciones de las personas facilitadoras se deben realizan en el momento por medio de palabras de motivación, refuerzos positivos, correcciones motrices, claves motrices, entre otros.

Cada momento de aprendizaje es planificado a priori por las personas facilitadoras del proyecto, según la Tabla 1 Progresión de las áreas del desarrollo humano, de acuerdo con la etapa de vida y el modelo del Proyecto Psicomotricidad e Intervención. Es importante señalar que la Tabla 1 fue desarrollada por el equipo de trabajo de este proyecto tomando como fundamentos la pirámide de desarrollo de Lázaro y Berruezo (2009), las teorías del desarrollo humano y el modelo teórico del desarrollo motor (Gallahue, Ozmun y Goodway, 2011).

A su vez, es necesario aclarar que en cada momento de aprendizaje se realizan una variedad de actividades, juegos, vivencias, acciones orientas en el descubrimiento guiado y el Master Motivacional Climate, métodos que permiten una continua exploración y aprendizaje del infante según sus características y necesidades. 
Tabla 1

Progresión de las áreas del desarrollo humano según etapa de

vida y el modelo del Proyecto Psicomotricidad e Intervención

\begin{tabular}{|c|c|c|c|}
\hline \multirow{2}{*}{$\begin{array}{c}\text { Área por } \\
\text { desarrollar }\end{array}$} & \multicolumn{3}{|c|}{ Etapas de vida } \\
\hline & 2 y 3 años & 4 y 5 años & 6 y 7 años \\
\hline $\begin{array}{l}\text { Motora } \\
\text { gruesa }\end{array}$ & $\begin{array}{l}\text { Esquema corporal y } \\
\text { acciones del cuerpo } \\
\text { Tono y relajación } \\
\text { Conciencia } \\
\text { respiración } \\
\text { Destrezas } \\
\text { rudimentarias }\end{array}$ & $\begin{array}{l}\text { Equilibrio y } \\
\text { coordinación dinámica } \\
\text { general } \\
\text { Destrezas básicas } \\
\text { Conciencia espacial } \\
\text { Direccionalidad } \\
\text { Imagen Corporal }\end{array}$ & $\begin{array}{l}\text { Coordinación } \\
\text { visomotriz } \\
\text { Destrezas básicas } \\
\text { Conciencia lateral- } \\
\text { conceptual }\end{array}$ \\
\hline Motora fina & $\begin{array}{l}\text { Agarrar } \\
\text { Prensar } \\
\text { Poner }\end{array}$ & Formas y tamaños & Trazos de escritura \\
\hline Cognitiva & $\begin{array}{l}\text { Desarrollo lógico- } \\
\text { matemático } \\
\text { Ordenamiento }\end{array}$ & $\begin{array}{l}\text { Desarrollo lógico- } \\
\text { matemático } \\
\text { Identificación y } \\
\text { clasificación }\end{array}$ & $\begin{array}{l}\text { Desarrollo lógico- } \\
\text { matemático } \\
\text { Operaciones } \\
\text { matemáticas }\end{array}$ \\
\hline Perceptual & $\begin{array}{l}\text { Sentidos individuales } \\
\text { Percepción propio } \\
\text { cuerpo }\end{array}$ & $\begin{array}{l}\text { Capacidad de integración } \\
\text { sensorial }\end{array}$ & $\begin{array}{l}\text { Propiocepción } \\
\text { Ajuste y control } \\
\text { postural }\end{array}$ \\
\hline Social & $\begin{array}{l}\text { Participación activa } \\
\text { de la familia (área } \\
\text { acuática y aire libre) } \\
\text { Relación con pares } \\
\text { (área salón) }\end{array}$ & $\begin{array}{l}\text { Participación activa de la } \\
\text { familia } \\
\text { Relación con pares (área } \\
\text { salón) } \\
\text { Relación con persona } \\
\text { mayores a su edad }\end{array}$ & $\begin{array}{l}\text { Participación activa } \\
\text { de la familia (área } \\
\text { acuática y aire libre) } \\
\text { Relación con pares } \\
\text { (área salón) } \\
\text { Trabajo equipo }\end{array}$ \\
\hline Autonomía & Autorregulación & Autonomía & Autonomía \\
\hline $\begin{array}{l}\text { Planificación } \\
\text { motriz } \\
\text { (praxis) }\end{array}$ & Movimiento simple & Movimiento compuesto & Movimiento múltiple \\
\hline \multirow[t]{3}{*}{$\begin{array}{l}\text { Eje } \\
\text { trasversal }\end{array}$} & $\begin{array}{l}\text { Conciencia } \\
\text { respiración }\end{array}$ & Conciencia respiración & $\begin{array}{l}\text { Conciencia } \\
\text { respiración }\end{array}$ \\
\hline & Destrezas de lenguaje & Destrezas de lenguaje & $\begin{array}{l}\text { Destrezas de } \\
\text { lenguaje }\end{array}$ \\
\hline & $\begin{array}{l}\text { Demostración de } \\
\text { emociones } \\
\text { (identificar-expresión- } \\
\text { manejo) }\end{array}$ & $\begin{array}{l}\text { Demostración de } \\
\text { emociones } \\
\text { (identificar-expresión- } \\
\text { manejo) }\end{array}$ & $\begin{array}{l}\text { Demostración de } \\
\text { emociones } \\
\text { (identificar- } \\
\text { expresión-manejo) }\end{array}$ \\
\hline
\end{tabular}

Nota: elaboración propia.

En el proyecto, su personal de apoyo efectúa una realimentación constante (feedback) en torno a: las acciones de los niños y las niñas, la importancia del seguimiento de instrucciones, la atención a los límites, la expresión de emociones y la promoción de valores como el respeto, solidaridad, honradez, amistad, tolerancia, compartir, entre muchos otros.

Cuando la población infantil se encuentra en el momento de salón, se aprovecha para que las personas familiares o encargadas reciban capacitación y espacios para la reflexión. En la capacitación, el padre, 
la madre o el encargado serán partícipes en talleres mensuales en temas como: promoción de estilos de vida saludables, elaboración de loncheras y meriendas saludables, manejo de límites con los hijos e hijas, problemas de conducta, práctica de valores, adherencia a la actividad física de un adulto padre o madre, importancia de la actividad física en la edad madura, áreas del desarrollo humano, contribución del movimiento y el juego en el formación integral de esta población, entre otros temas. Estos talleres son efectuados por personal del equipo de PSICOMI, entre ellos profesionales en Educación Física, Deporte y Recreación, Promoción de la Salud, Psicopedagogía, Psicología y otros profesionales invitados.

Además, como complemento del proyecto los padres, las madres y los encargados se integran a actividades de ejercico físico dirigido por profesionales en el área de la Promoción de la Salud Física, con el fin de sensibilizar a la familia de la importancia de tener estilos de vida saludables y, a su vez, al ser partícipe de esta viviencia, refuerza, acompaña y apoya la creación de hábitos de vida desde la infancia.

\section{Resultados}

\section{Puesta en práctica del modelo}

Para poder implementar este modelo, se plantearon una serie de pautas administrativas esenciales para la puesta en práctica del mismo. En la primera sesión, se invita a la familia a participar en una sesión gratuita para que conozcan y vivencien la metodología. Seguidamente, se les da información acerca de requisitos, disposiciones, costos, horarios, entre otros. Una vez que la familia ingresa al programa se le entrega la siguiente documentación:

a) Material de información sobre el programa.

b) Hoja de requisitos de cada espacio, vestuario, reglamento del uso de la piscina y áreas verdes de CIEMHCAVI, recomendaciones de vestimenta, uso de bloqueador, elaboración de meriendas saludables.

c) Ficha de identificación del niño o niña.

d) Consentimiento de la persona encargada del niño o niña participante en el programa, donde autorizan la asistencia a las sesiones, la aplicación de evaluaciones y otras actividades que el programa promueva.

En una segunda sesión, se le aplica al infante una prueba de desarrollo motor. Seguidamente, el psicopedagogo del proyecto revisa el expediente con la totalidad de la información brindada por la familia y los resultados de la prueba de desarrollo motor, para en la sesión siguiente informarle a la familia los resultados encontrados, en qué punto de partida se encuentra el niño o la niña y el grupo que le corresponde. Cabe señalar que el grupo que se le asigna al niño o niña no siempre corresponde con su edad cronológica.

Una vez concluida esta parte, el personal instructor elabora los planes mensuales y semestrales, los cuales se van ajustando según las necesidades mostradas por la población infantil. Como parte del seguimiento al trabajo con esta población, el psicopedagogo realiza sesiones de entrevista psicopedagógica durante el año que le permitan dar recomendaciones o indicaciones tanto para el personal del programa como a las familias. También, de ser necesario, se realizan evaluaciones psicopedagógicas específicas al niño o la niña que lo requiera. Además, anualmente se elabora un informe psicopedagógico que se incorpora al expediente del infante en el programa, se entrega una copia a la familia y, si es necesario, se envía a la institución educativa correspondiente.

\section{Fortalezas detectadas en la aplicación del modelo}

Durante los cuatro años de aplicación del proyecto, se han detectado las siguientes fortalezas en el modelo:

1. Permite tener un mejor control de las áreas y temáticas a desarrollar durante los diferentes espacios de aprendizaje, lo que favorece el engranaje del trabajo entre todas las personas actoras. 
2. Permite que el trabajo del personal que colabora en el proyecto siga un ordenamiento lógico, metodológico, pedagógico y no estímulos aislados. Asimismo, se ha convertido en un espacio de formación de estudiantes para sus prácticas profesionales, prácticas docentes, prácticas de cursos e investigaciones.

3. Al contar con dos espacios de aprendizaje donde se requiere la participación de la persona a cargo del niño o la niña, la relaciones entre estos se han visto enriquecidas, ya que en algunos casos es el único espacio donde padre-madre-encargado comparte tiempo con el hijo o la hija. A su vez, esta participación ha permitido la realimentación del infante al adulto y viceversa. De la misma manera, ha favorecido la formación del padre-madre-encargado en materia de necesidades y desarrollo del infante.

4. $\quad$ Permite que el padre-madre-encargado aprenda a jugar y valore la importancia de este en el desarrollo del niño y la niña.

5. Al haber espacios de aprendizaje donde se propician las interacciones personales múltiples, (guías, infante, pares o niños y niñas mayores, persona encargada) el niño o la niña aprende a relacionarse con personas de todas las edades y logra crear entornos de aprendizaje que les permite identificar sus propias posibilidades y a su vez le permite aprender del modelaje de otros.

6. El niño o la niña es en todo el momento el que dicta el avance metodológico según su edad madurativa y cronológica, por lo que, se han reportado cambios en esta población que han sido perdurables en el tiempo en cuanto actitudes, destrezas, habilidades, tareas y hábitos, los cuales se han logrado transferir no solo en el ambiente del proyecto sino también a nivel del hogar, del centro educativo u otros entornos donde el niño o la niña se desenvuelven.

7. Permite exportar el proyecto a cualquier otra zona geográfica del país, ya que están descritas de forma clara y precisa las pautas metodológicas del mismo.

\section{Oportunidades que brinda el Modelo de Intervención del Proyecto Psicomotricidad e Intervención}

A través de las perspectivas de las personas encargadas de los niños y las niñas participantes del proyecto, los comentarios mismos de la niñez beneficiada y las observaciones efectuadas por el equipo de trabajo, se han detectado las siguientes oportunidades de cambios sociales o transformaciones de los sujetos y sus relaciones desde la experiencia:

1. Se ha fomentado en la población participante hábitos y actitudes como levantarse temprano, mayor responsabilidad para hacer sus deberes, disfrute de la actividad física, mejoramiento de la conducta escolar, entre otros.

2. Uno de los principales cambios que han observado las personas encargadas con respecto a sus hijos o hijas es en la seguridad en sí mismos, el aumento de independencia, la disminución de temores y la capacidad para estar solo o sin sus padres.

3. Se ha reportado disminución de la timidez, un incremento en las expresiones emocionales, mayor seguridad al relacionarse con otros, mayores niveles de sociabilidad con personas y con animales.

4. La familia señala sentirse feliz, satisfecha con el comportamiento que va adquiriendo su hija o hijo durante la participación del proyecto.

5. Se destacan mejoras en el área perceptual, las destrezas motoras básicas y deportivas, el desempeño en las clases de educación física y actividades recreativas.

6. Se ha mejorado las relaciones con pares, el manejo y control de impulsos, una mayor tolerancia a la frustración y mejor manejo y expresión de emociones.

7. Mejoras en el trabajo en equipo, lo que según las personas encargadas del niño o la niña les permiten una mayor actitud en el aprendizaje y el rendimiento escolar.

8. Incremento en la atención y un aumento en el entendimiento de los límites, se encuentra más tranquilos, más obedientes.

9. El niño o la niña ha logrado procesos de autorregulación de su conducta y de su aprendizaje, al punto que corrige a sus superiores o pares para que logren ejecutar acciones de manera exitosa. 


\section{Conclusiones y recomendaciones futuras}

El proyecto Psicomotricidad e Intervención desde su modelo propicia el desarrollo integral de la población infantil, por medio de vivencias que permiten la adquisición y mejoramiento de destrezas y habilidades sociales, emocionales, cognitivas y motrices, en cada infante y sus familias, para que construyan desde su realidad herramientas que les permitan resolver de una manera asertiva los retos que le presenta la vida. El infante desarrolla una autorregulación y una autonomía, influyendo en procesos de seguridad, autoestima y autoconcepto, los cuales no solo contribuyen en su bienestar familiar sino en otros ambientes como la escuela.

El constructivismo y las teorías de aprendizaje que fundamentan el modelo de intervención permiten que el niño y la niña sea el protagonista desde su propio aprendizaje, y el resto de las personas actoras contribuyen y apoyan de manera activa esa construcción de conocimiento. Contar con la participación de las familias en dos de los tres momentos fomenta el lazo socioafectivo entre el infante y sus familias, creando un ambiente de satisfacción y un sentimiento de bienestar en la vida familiar.

El modelo PSICOMI se representa como una alternativa para que el padre, madre, encargado o profesionales de diferentes áreas orienten la formación, educación, programas o currículo, y potencien, a través de la motricidad, estrategias y actividades para permear el desarrollo integral de cada infante y sus estilos de vida saludables desde edades tempranas.

Como recomendaciones futuras, se quiere la elaboración de una investigación-acción que permita valorar de manera cuantitativa el impacto del modelo en las diferentes áreas del desarrollo del infante. A su vez, es necesario realizar mayores investigaciones utilizando este modelo de intervención en otras comunidades o culturas para comparar sus alcances. Asimismo, este modelo podría ser replicable en otros entornos tanto a nivel nacional como internacional, con el fin de valorar sus impacto y contribución a la sociedad.

\section{Referencias}

Álvarez, C. (2015). Factores que influencian un estilo de vida sedentario en las estudiantes de cuarto ciclo de los colegios públicos. Revista MHSalud, 12(1). Recuperado de https://doi.org/10.15359/ mhs.12-1.1

Álvarez, C. (2016a). Efectividad del programa ecológico "Saltando por su salud" en la promoción de la actividad física y la auto-eficacia en niños y niñas escolares de tercer grado. Ensayos Pedagógicos, 11(1). Recuperado de http://www.revistas.una.ac.cr/index.php/ensayospedagogicos/ article/view/8473/0

Álvarez, C. (2016b). Entendiendo los factores que determinan la actividad física en el entorno escolar desde la perspectiva de los niños y niñas. Revista MHSalud, 13(1). Recuperado de https:// doi.org/10.15359/mhs.13-1.2

Ames, C. (1992a). Achievement goals, motivational climate, and motivational processes. In G. C. Roberts (Ed.), Motivation in Sport and Exercise (pp. 161-176). Champaign, IL: Human Kinetics.

Ames, C. (1992b). Classroom: Goals, structures, and student motivation. Journal of Educational Psychology, 84, 409-414. doi: https://doi.org/10.1037/0022-0663.84.3.261

Bruner, J. S. (1995). Desarrollo congnitivo y educación. Selección de textos por Jesús Palacios (2da. Ed.). España: Ediciones Morata, S. L.

Díaz-Barriga Arceo, F. y Hernández Rojas, G. (2010). Estrategias docentes para un aprendizaje significativo: una interpretación constructivista (3ra ed.). México: McGraw Hill. 
Fernández Rivas, M. y Espada Mateos, M. (2016). Formación inicial y percepción del profesorado sobre los estilos de enseñanza en Educación Física. Retos, 31, 69-75. Recuperado de https://recyt.fecyt.es/ index.php/retos/article/view/49024

Gallahue, D. L., Ozmun, J. C., \& Goodway, J. D. (2011). Understanding motor development: Infants, children, adolescents, adults (7th. ed.). USA: McGraw Hill.

Herrera, E., Araya, G., Fernández, H., Morera, M., y Fonseca, H. (2015). El diagnóstico temprano de niños y niñas con riesgo académico mediante un sistema de diagnóstico perceptual-motor: Estudio retrospectivo longitudinal de evidencias de su efectividad. Revista Electrónica Educare, 19(3), 1-14. doi: de https://doi.org/10.15359/ree.19-3.1

Herrera, E., Delgado, L., Fonseca, H., y Vargas, P. (2012). Factores socio-afectivos relacionados con las dificultades escolares en niñas y niños "estrella" del programa psicomotricidad e intervención. Revista MHSalud, 9(1). Recuperado de http://www.revistas.una.ac.cr/index.php/mhsalud/issue/ view/362

Herrera-González, E., Morera-Castro, M., Vargas-Araya, G, Fonseca-Schmidt, H. y Vargas-Ramírez, P. (2017). Análisis del estado físico y psicológico de la población escolar del cantón central de Heredia, Costa Rica. Revista MHSalud, 13(2). doi: http://dx.doi.org/10.15359/mhs.13-2.7

Lázaro, A. y Berruezo, P. (2009). La pirámide del desarrollo humano. Revista Iberomericana de Psicomotricidad y Técnicas Corporales, 9(34), 15-42. Recuperado de http:// www.colegiogloriafuertes.es/articulos/articulo2piramide.pdf

Ministerio de Educación Pública [MEP]. (2002). Consideraciones y Lineamientos para el Desarrollo de la Práctica Pedagógica en el Nivel Preescolar. San José, Costa Rica: Talleres M.E.P.

Monereo, C., Badía, A., Baixeras, M. V., Boadas, E., Castelló, M., Guevara, I., ... y Sebastiani, E. M. (2008). Ser estratégico y autónomo aprendiendo (3ra. Ed). Barcelona, España: Editorial GRAÓ.

Mosston, M. y Ashworth, S. (1986). La enseñanza de la Educación Física. Barcelona: Editorial Hispano Europea, S. A.

Programa Estado de la Nación (2005). Estado de la Educación. Primer Informe, San José, Proyecto Estado de la Nación. Recuperado de http://www.estadonacion.or.cr/informe-i-estado-educacion

Programa Estado de la Nación (2008a). Estado de la Educación. Segundo Informe, San José, Proyecto Estado de la Nación. Recuperado de http://estadonacion.or.cr/files/biblioteca_virtual/ educacion/002/Info2edusinopsis.pdf

Programa Estado de la Nación (2011). Estado de la Educación. Tercer Informe, San José, Proyecto Estado de la Nación. Recuperado de http://www.estadonacion.or.cr/files/biblioteca_virtual/educacion/003/ Parte_1_Capitulo_2-edu03.pdf

Programa Estado de la Nación (2013). Estado de la Educación. Cuarto Informe, San José, Proyecto Estado de la Nación. Recuperado de http://www.estadonacion.or.cr/files/biblioteca_virtual/ educacion/004/6-Cap-2.pdf

Programa Estado de la Nación (2015). Estado de la Educación. Quinto Informe, San José, Proyecto Estado de la Nación. Recuperado de http://www.estadonacion.or.cr/educacion2015/assets/cap-2ee-2015.pdf

Programa Estado de la Nación. (2002). Estado de la Nación en Desarrollo Humano Sostenible. VIII Informe, San José, Proyecto Estado de la Nación. Recuperado de http://estadonacion.or.cr/files/ biblioteca_virtual/008/info8cap2.pdf

Programa Estado de la Nación (2006). Estado de la Nación en Desarrollo Humano Sostenible. XII Informe, San José, Proyecto Estado de la Nación. Recuperado de http://estadonacion.or.cr/files/ biblioteca_virtual/012/info12cap2.pdf 
Programa Estado de la Nación (2008b). Estado de la Nación en Desarrollo Humano Sostenible. XIV Informe, San José, Proyecto Estado de la Nación. Recuperado de http://estadonacion.or.cr/files/ prensa/informe-XIV/cap2_equidad_14.pdf

Programa Estado de la Nación (2010). Estado de la Nación en Desarrollo Humano Sostenible. XVI Informe, San José, Proyecto Estado de la Nación. Recuperado de http://estadonacion.or.cr/files/ biblioteca_virtual/016/2-CAP-2-informe16.pdf

Trilla, J., Cano, E., Carretero, M., Escofer, A., Fairstein, F., Fernández Fernández, J. A., ... y Vila, I. (2007). El legado pedagógico del siglo XX para la escuela del siglo XXI. Barcelona, España: Editorial GRAÓ.

Valentini, N. C., Rudisill, M. E., \& Goodway, J. D. (1999a). Mastery climate: Children in charge of their own learning. Teaching Elementary Physical Education, 10, 6-10.

Valentini, N. C., Rudisill, M. E., \& Goodway, J. D. (1999b). Incorporating a Mastery Climate into Physical Education: It's Developmentally Appropriate! Journal of Physical Education, Recreation \& Dance, 70(7), 28-32. doi: https://doi.org/10.1080/07303084.1999.10605683

Valentini, N. C., \& Rudisill, M. E. (2004). Effectiveness of an inclusive mastery climate intervention on the motor skill development of children with and without disabilities. Adapted Physical Activity Quarterly, 21(4), 330-347. doi: https://doi.org/10.1123/apaq.21.4.330

Wall, S., \& Rudisill, M. E. (2002). Active Start: A Statement of Physical Activity Guidelines for Children Birth to Five Years. Journal: Teaching Elementary Physical Education: Preschool Community Collaborations.

Wall, S., \& Rudisill, M. (2004). Meeeting active start guidelines: Toddlers. Teaching Elementary Physical Education: Preschool Community Collaborations.

Woodburn, S., Rodríguez, F. y Boschini, C. (1997). El desarrollo motor en niños: Prueba de desarrollo motor-Universidad Nacional (PDM-UNA). Heredia y San José, Costa Rica: EUNA y EUCR.

Woodburn, S., Camacho, T., Boschini, C., Fernández, H. y Rodríguez, F. (1991). Prueba KTK, adaptada (Prueba de coordinación corporal para niños): Manual instructivo. Heredia, Costa Rica: Escuela Ciencias del Deporte, Universidad Nacional.

Woodburn, S. (1997a). El desarrollo gestalt en niños: Prueba de Anton Brenner, adaptada (PGAB). Heredia, Costa Rica: Escuela Ciencias del Deporte, Universidad Nacional. Manuscrito inédito.

Woodburn, S. (1997b). La madurez intelectual en niños: La prueba Goodenough-Harris (PGH). Heredia, Costa Rica: Escuela Ciencias del Deporte, Universidad Nacional. Manuscrito inédito.

Woodburn, S. y Boschini, C. (1997). Los problemas de aprendizaje en niños: Test de la Escuela Meeting Street (Adaptado). San José y Heredia, Costa Rica: Editorial de la Universidad de Meeting Street.

Woodburn, S., Boschini, C. y Fernández, H. (1997). La imagen corporal en niños: Prueba para diagnóstico de imagen corporal (PDIC). San José y Heredia, Costa Rica: EUCR.

Woodburn, S., Sisfontes, P. y Fernández, H. (1998). Valoración y actividades interactivas para la educación psicomotriz. Heredia, Costa Rica: Editorial Universidad de Costa Rica. 